\title{
Surfaces
}

\section{Geoffrey Bennington, Jacques Derrida (avec Jacques Derrida, Circonfession), (Paris: Seuil, 1991)}

\section{Michel Peterson}

Volume 2, 1992

URI : https://id.erudit.org/iderudit/1065244ar

DOI : https://doi.org/10.7202/1065244ar

Aller au sommaire du numéro

Éditeur(s)

Les Presses de l’Université de Montréal

ISSN

1188-2492 (imprimé)

1200-5320 (numérique)

Découvrir la revue

Citer ce compte rendu

Peterson, M. (1992). Compte rendu de [Geoffrey Bennington, Jacques Derrida (avec Jacques Derrida, Circonfession), (Paris: Seuil, 1991)]. Surfaces, 2.

https://doi.org/10.7202/1065244ar

Ce document est protégé par la loi sur le droit d'auteur. L'utilisation des services d'Érudit (y compris la reproduction) est assujettie à sa politique d'utilisation que vous pouvez consulter en ligne.

https://apropos.erudit.org/fr/usagers/politique-dutilisation/ 


\section{COMPTE RENDU \\ GEOFFREY BENNINGTON: \\ JACQUES DERRIDA}

Michel Peterson

Geoffrey Bennington, Jacques Derrida (avec Jacques Derrida,
Circonfession), (Paris: Seuil, 1991).

Jacques Derrida se présente comme une série de textes complexes et denses qui organisent en un faisceau conceptuel les principaux motifs (traduction, littérature, don, signature, féminité, clôture, trace, etc.) allégués et tissés par Derrida depuis plusieurs années au hasard de ses lectures. Élaboré selon le modèle d'un logiciel interactif (le "Derridiciel"), l'ouvrage présente les enjeux, les exigences et les ressources de la déconstruction, lesquels sont lus non dans le but de proposer une vérité mais bien dans celui, plus difficile, de faire surgir la singularité d'une pensée qui se déploie dans les dimensions de la responsabilité et de la nécessité, c'est-à-dire d'une éthique qui ne fonctionne pas selon les règles positives de l'intérêt objectif.

Trois textes. Dans le premier : "Derridabase", Bennington traverse l'"oeuvre" de Derrida afin d'en fournir les données. Dans le second : "Circonfession", Derrida rédige un texte lié par contrat au premier en exigeant sa présence. Enfin, "Actes (la loi du genre)" procède à la constitution historique du sujet Derrida en établissant un curriculum vitae qui déjoue la linéarité temporelle. La thèse de Bennington (s'agit-il bien d'une thèse?) est la suivante : l'apport de Derrida à l'histoire de la philosophie consiste dans le fait que le penseur de la différance rend immaîtrisable l'opposition entre l'empirique et le transcendantal - non-opposition reconnue ici sous le nom de quasitranscendantalité (terme emprunté, via Derrida lui-même, à Rodolphe Gasché, l'un des meilleurs commentateurs de Derrida).

Flanquant la lecture de Bennington/Derrida et renouant avec Glas, "Circonfession" est composé de 59 périodes qui excèdent le "théologiciel" 
construit par Bennington, en plus d'offrir une "traduction" des Confessions de Saint Augustin. À travers une "génération de propositions", Derrida élabore un texte qui tourne autour du problème de la circoncision et de la mort de sa mère. Cette biographie constitue un commentaire du judaïsme dont la beauté et la rigueur répondent à l'appel impérieux de Bennington.

Commandé par le souci de montrer le fonctionnement du système général de la pensée derridienne (pour autant que l'on puisse ici parler de "système" -- ce qui est on ne peut plus douteux), cet ouvrage offre en outre une riche bibliographie et de nombreuses photographies. Inutile toutefois de chercher à savoir à qui est destiné ce livre, à qui il est offert, et à partir d'où il advient. De même qu'il aurait été impossible pour Bennington d'exécuter une lecture dite derridienne non pas de l'oeuvre derridienne mais de Derrida lui-même, de même il serait dans ce cadre-ci rigoureusement insensé de tenter d'effectuer une lecture benningtonienne de Bennington. De l'envoi qui pointe la clôture du texte de Bennington, extrayons donc simplement ceci : "[...] nous avons dit les limites du commentaire et de l'interprétation en nous limitant au commentaire et un peu (très peu) d'interprétation. Double bind où notre fidélité absolue a été l'infidélité même." (p. 292) Double bind en effet que ces chiasmes textuels où s'engendrent des lectures vouées à l'in-expérience perpétuelle et monumentale de la lecture. Déjouées par ce qui s'appelle l'illégalité intrinsèque de la loi, de toute loi du genre, de toute loi de la Loi, des constellations apparaissent entre le fini et l'infini qui mettent en échec la distance herméneutique et éprouvent la possibilité d'une lecture qui se veut non-commentaire.

L'interprète ne se conçoit alors que dans la perspective souveraine de sa mort impossible. Expérience et lecture se jouent dans la non-appartenance et dans toutes sortes de tentatives innommables pour sortir enfin du phénoménal qui dicte l'impératif catégorique. Jeu sans jeu du "quasi", de l'"antre" et, surtout, de la chance. "C'est pourquoi ce livre ne vous servira en rien, à vous autres, à vous, l'autre, et n'aura été qu'un prétexte dérobé pour y inscrire ma propre signature derrière, dans son dos." (p. 292)

On pense, immanquablement, à l'étrange et éblouissante fin du Pour un Malherbe de Francis Ponge, le poète résistant (plus encore, quoi qu'on en dise, que Mallarmé, Claudel ou Valéry) auquel Jacques Derrida, sur le dos de son nom, a consacré quelques-unes de ses plus belles pages à propos de l'inscription de la signature dans les passages du cadre. Exposant les motifs qui poussent un individu, quel qu'il soit, à acheter un livre, Francis Ponge prétend que l'un d'eux est de procurer à son âme un moyen de transport, c'est-à-dire une métaphore. Il s'agit, dans ce trajet balisé par un procès consistant à faire "porter sur" (épi) un nom un autre qui conserve son étrangeté -- par exemple, faire porter sur Francis Ponge le nom Francis Ponge ou celui de Geoffrey Bennington sur Jacques Derrida ou... --, de se déplacer et de déplacer le langage et, partant, le déplacement lui-même. D'où, dans le texte de Ponge, la question du changement de vitesse et l'apparition d'une vitesse critique, laquelle définit tout aussi bien le rythme qu'impose Jacques Derrida. Lecture du Pour un Malherbe : 
Le grand art est de prendre le lecteur de plain-pied (sans qu'il s'en aperçoive et s'effraye), et de l'enlever aussitôt.

Bien. Dès la première phrase de Bennington, nous sommes enlevés : "Il faudrait, donc, montrer en quoi Derrida est un "contemporain". Surprise, donc, ou incrédulité, ou crise de légitimité? Inutile d'insister, nous sommes instantanément saisis, nous avons compris. Continuons Ponge, le manifeste se désignera lui-même dans la non-propriété :

L'attaque [nous sommes sur ce que Ponge appelle ailleurs le "pré"] a donc une grande importance. Le saisissement doit être immédiat et l'enlèvement réel : il ne faut pas que le lecteur bute, bronche, s'effraye, hésite, ait l'impression non peut-être de ne pas comprendre, mais de n'être pas compris, concerné.

Pour un enlèvement, un intérêt réels.

\author{
Michel Peterson
}

Université de Montréal

Surface Page d'Acceuil/Home Page 